\title{
Reciprocal Interaction of Dendrite Geometry and Nuclear Calcium-VEGFD Signaling Gates Memory Consolidation and Extinction
}

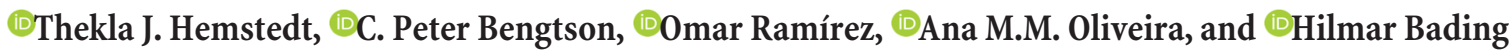 \\ Department of Neurobiology, Interdisciplinary Center for Neurosciences, Heidelberg University, 69120 Heidelberg, Germany
}

\begin{abstract}
Nuclear calcium is an important signaling end point in synaptic excitation-transcription coupling that is critical for long-term neuroadaptations. Here, we show that nuclear calcium acting via a target gene, VEGFD, is required for hippocampus-dependent fear memory consolidation and extinction in mice. Nuclear calcium-VEGFD signaling upholds the structural integrity and complexity of the dendritic arbor of CA1 neurons that renders those cells permissive for the efficient generation of synaptic input-evoked nuclear calcium transients driving the expression of plasticity-related genes. Therefore, the gating of memory functions rests on the reciprocally reinforcing maintenance of an intact dendrite geometry and a functional synapse-to-nucleus communication axis. In psychiatric and neurodegenerative disorders, therapeutic application of VEGFD may help to stabilize dendritic structures and network connectivity, which may prevent cognitive decline and could boost the efficacy of extinction-based exposure therapies.
\end{abstract}

Key words: calcium signaling; dendrite morphology; gene expression; memory function; nuclear calcium; VEGFD

\section{Significance Statement}

This study uncovers a reciprocal relationship between dendrite geometry, the ability to generate nuclear calcium transients in response to synaptic inputs, and the subsequent induction of expression of plasticity-related and dendritic structure-preserving genes. Insufficient nuclear calcium signaling in CA1 hippocampal neurons and, consequently, reduced expression of the nuclear calcium target gene VEGFD, a dendrite maintenance factor, leads to reduced-complexity basal dendrites of CA1 neurons, which severely compromises the animals' consolidation of both memory and extinction memory. The structure-protective function of VEGFD may prove beneficial in psychiatric disorders as well as neurodegenerative and aging-related conditions that are associated with loss of neuronal structures, dysfunctional excitation-transcription coupling, and cognitive decline.

\section{Introduction}

The formation and extinction of fear, an evolutionarily conserved emotion, is important for the survival of an animal. Dys-

Received July 22, 2016; revised May 8, 2017; accepted May 9, 2017.

Author contributions:T.J.H., C.P.B., O.R., A.M.M.O., and H.B. designed research; T.J.H., C.P.B., and 0.R. performed research; T.J.H., C.P.B., O.R., A.M.M.O., and H.B. analyzed data; T.J.H., C.P.B., O.R., A.M.M.O., and H.B. wrote the paper.

This work was supported by the European Research Council (Advanced Grant to H.B.), the Deutsche Forschungsgemeinschaft Sonderforschungsbereich (SFB) 636 and SFB 1134, Conicyt Becas Chile-Postdoctorado 74150080 (0.R.), and the Hartmut Hoffmann-Berling International Graduate School of Molecular and Cellular Biology of Heidelberg University. H.B. and A.M.M.O. are members of the Excellence Cluster CellNetworks at Heidelberg University. We thank I. Bünzli-Ehret for the preparation of hippocampal cultures and Daniela Mauceri for providing images of neurons for fractal analysis and for discussions. Images of Golgi-impregnated tissues were taken at the Nikon Imaging Center at Heidelberg University.

The authors declare no competing financial interests.

Correspondence should be addressed to Hilmar Bading, Department of Neurobiology, Interdisciplinary Center for Neurosciences, Heidelberg University, Im Neuenheimer Feld 364, 69120 Heidelberg, Germany. E-mail: bading@nbio.uni-heidelberg.de.

DOI:10.1523/JNEUROSCI.2345-16.2017

Copyright $\odot 2017$ the authors $\quad 0270-6474 / 17 / 376946-10 \$ 15.00 / 0$ functional responses to fear-relevant and fear-irrelevant contexts and cues lead to maladaptations that may result in the development of anxiety disorders. The establishment of an association between a previously neutral cue and a fearful experience induces fear memory formation. These memories are expressed as a fearful response upon the presentation of the cue even in the absence of the threatening situation. A repeated experience of a fearevoking cue in the absence of the aversive stimulus may lead to a loss of that fear, which is known as fear extinction. Several therapeutic interventions for anxiety disorders are based on fear extinction, yet our understanding of the cellular and molecular mechanisms of fear extinction is far from complete. It is widely accepted that fear extinction generates a new memory in which a particular cue is no longer associated with the aversive stimulus. This new memory, which requires synaptic-activity-dependent gene transcription for its consolidation (Barondes and Cohen, 1968; Katche et al., 2013), suppresses rather than erases the old memory (Bouton, 2004). Nuclear calcium represents an important signaling end point in excitation-transcription coupling that 
drives gene expression programs needed for behavioral adaptations including memory formation (West and Greenberg, 2011; Bading, 2013). One of the key targets of nuclear calcium signaling is VEGFD, a dendrite maintenance factor critical for memory formation (Mauceri et al., 2011, 2015). In this study, we investigated whether structural integrity regulated by nuclear calciumVEGFD signaling is required for excitation-transcription coupling and fear memory formation and extinction.

\section{Materials and Methods}

Mice. We used male C57Bl/6N mice (Charles River Laboratories) from 8 to 12 weeks of age. The mice were group-housed (maximum four per cage) on a $12 \mathrm{~h}$ light/dark cycle with food and water ad libidum. Behavioral experiments were done during the light cycle. Mice that were sick and/or injured from cage-mate fighting were excluded from the study. All procedures were performed according to the German guidelines for the care and use of laboratory animals and with the European Community Council Directive 86/609/EEC.

Recombinant adeno-associated viruses ( $r A A V s)$. Viral particles were produced and purified as described previously (Zhang et al., 2007). To drive the expression of PV.NLS-mCherry, mCherry.NLS and HA-tagged VEGFD, we used a viral vector containing a CMV/chicken $\beta$ actinpromoter. For expression of shRNAs, an rAAV vector containing the U6 promoter for shRNA expression and a CaMKII promoter driving mCherry expression was used (Mauceri et al., 2011).

Stereotaxic surgery. rAAVs were injected into the dorsal hippocampus using the following coordinates relative to bregma: $-2 \mathrm{~mm}$ anteroposterior, $\pm 1.5 \mathrm{~mm}$ mediolateral, $-1.7,-1.9$, and $-2.1 \mathrm{~mm}$ dorsoventral. A total volume of $1.5 \mu \mathrm{l}$ was injected per hemisphere. For single infection, a 2:1 mixture of viral solution and $20 \%$ mannitol was used. In experiments with double infection, a mixture of 1:1:1 for viral solutions and mannitol was used; rAAV-mC.NLS- or rAAV-PV.NLS- $m C$-only injections were done using a mixture of 1:1:1 saline:virus:mannitol. The injection speed was $200 \mathrm{nl} / \mathrm{min}$ through a $33 \mathrm{Ga}$ needle. The needle was left for $60 \mathrm{~s}$ at each spot to allow the fluid to diffuse. Infection rate was validated for each animal. Animals with low viral expression were excluded from the analysis.

Morphometric analysis. Golgi impregnation was done using the Rapid Golgi Stain Kit (FD Neuro Technologies) according to the manufacturer's protocol. $Z$-stacks of Golgi-stained CA1 neurons were imported in Fiji (Schindelin et al., 2012), calibrated, the basal dendrites traced manually using the simple neurite tracer plugin (Longair et al., 2011), and the total length computed. For Sholl analysis (Sholl, 1953), the shell interval was set to $5 \mu \mathrm{m}$ using a plugin available for Fiji. A minimum of four animals per condition were used for analysis.

Fractal analysis. Neuronal $z$-stack images were processed using the ImageJ plugin Simple Neurite Tracer (Longair et al., 2011) to obtain a traced $2 \mathrm{D}$ binary representation of the dendrites. Fractal dimension $\left(D_{\mathrm{B}}\right)$ calculation was done using the traced dendritic trees as input for the FracLac imageJ plugin, which applies the box counting method to calculate $D_{\mathrm{B}}$ from the ratio of the $\log$ of the number of new parts $N$, to the log of scale $C$ using the scaling rule $N=A C^{-D_{B}}$. The resulting $D_{\mathrm{B}}$ values are proportional to the complexity degree of the dendritic structures. Widefield $z$-stack images of the basal dendrites of Golgi-stained CA1 pyramidal neurons of mice stereotaxically injected into the dorsal hippocampus with rAAV- $m C . N L S$, rAAV-PV.NLS- $m C$, rAAV- $m C . N L S$ plus rAAV$V E G F D$, or rAAV-PV.NLS- $m C$ plus rAAV-VEGFD were analyzed. All confocal $z$-stack images of cultured mouse hippocampal neurons transfected with an expression vector for hrGFP or cotransfected with expression vectors for hrGFP plus either mC-NLS or PV.NLS-mC were from Mauceri et al., 2015.

Contextual fear conditioning (CFC). CFC experiments were done as described previously (Oliveira et al., 2012). Briefly, mice were placed into the conditioning chamber $(23 \times 23 \times 35 \mathrm{~cm}$; TSE Systems $)$ for $148 \mathrm{~s}$ before they received a mild $2 \mathrm{~s}$ foot shock $(0.5 \mathrm{~mA})$ and remained there for $30 \mathrm{~s}$ after shock termination. After 1 or $24 \mathrm{~h}$, mice were placed back into the conditioning chamber for $300 \mathrm{~s}$ and freezing, defined as the absence of movements except respiration, was measured manually and continuously.

Contextual fear memory extinction. Mice were conditioned as described under the section "Contextual fear conditioning" using a $0.7 \mathrm{~mA}$ foot shock. Two weeks after fear conditioning, mice were placed back into the conditioning chamber for $20 \mathrm{~min}$ in the absence of a foot shock for fear memory extinction training. Testing occurred $24 \mathrm{~h}$ later in a session that lasted $300 \mathrm{~s}$. During all sessions, freezing was scored manually and continuously. Because the initial freezing of individual mice within each group is highly variable in this protocol and because it is known that the dynamics in fear extinction vary considerably between experiments (Sananbenesi et al., 2007; Agis-Balboa et al., 2011; BahariJavan et al., 2012), the data were normalized to the first 5 min of extinction. This results in less variability and maintains the possibility of comparing independent experiments.

Spatial object recognition (SOR). SOR experiments were done as described previously (Oliveira et al., 2012). Briefly, mice were placed $4 \times 6$ min into an experimental apparatus with a visual cue on the wall. During the first $6 \mathrm{~min}$, the mice were habituated to the box without objects and, during the following 3 sessions, mice were allowed to explore 2 distinct objects. Between each session, mice were placed back into their home cages for $3 \mathrm{~min}$. Animals were tested during a $6 \mathrm{~min}$ session 1 or $24 \mathrm{~h}$ later for short-term and long-term memory, respectively. For the test session, one of the objects was displaced, the time the mice spent exploring each object was scored manually, and the preference for the displaced object was calculated.

Immunohistochemistry. Mice were perfused with 10\% formalin (SigmaAldrich) and postfixed in the same solution overnight. Brains were then placed into a solution containing $30 \%$ sucrose in $0.1 \mathrm{~m}$ PBS containing $0.04 \%$ thimerosal (Sigma-Aldrich) for cryoprotection. Brains were cut at a thickness of $30 \mu \mathrm{m}$ and collected and stored in PBS containing $0.04 \%$ thimerosal until further process. Slices were permeabilized with $0.1 \%$ Triton X-100 in PBS. They were blocked in $8 \%$ normal goat serum (NGS) with $0.3 \%$ Triton X-100 in PBS for 50 min at room temperature. Slices were incubated with the antibody against the HA tag (1:250, sc-805; Santa Cruz Biotechnology) diluted in PBS containing 2\% NGS and 0.3\% Triton X-100 overnight at $4^{\circ} \mathrm{C}$. Slices were incubated in the secondary antibody diluted in PBS for $2 \mathrm{~h}$ in the dark at room temperature. Sections were incubated in Hoechst 33258 (1:5000) for 5 min and mounted on glass slides.

Acute brain slice preparation. Brain slices were prepared from 11-weekold mice that had been stereotaxically injected 3 weeks prior with an rAAV. Mice were anesthetized with pentobarbital (Narcoren; Merial) injection intraperitoneally and perfused transcardially with slicing buffer containing the following (in $\mathrm{mM}$ ): $\mathrm{N}$-methyl-D-glucamine 93; $\mathrm{KCl}, 2.5$; $\mathrm{NaH}_{2} \mathrm{PO}_{4}, 1.2$; HEPES, 20; $\mathrm{NaHCO}_{3}, 30 ; \mathrm{CaCl}_{2}, 0.5 ; \mathrm{MgCl}_{2}, 7$; ascorbic acid, 5; thiourea, 2; sodium pyruvate, 3 ; $\mathrm{N}$-acetyl-cysteine, 10; glucose, 25 ; pH 7.3 with $\mathrm{HCl}$; gassed with $95 \% \mathrm{O}_{2}$ and $5 \% \mathrm{CO}_{2}$. After decapitation, the brain was rapidly removed and submerged in ice-cold slicing buffer. Three-hundred-micron-thick coronal slices of the dorsal hippocampus were cut at $0^{\circ} \mathrm{C}(\mathrm{CU} 65$ Cooling Unit, HM650V Vibratome; Microm) and transferred to a $32^{\circ} \mathrm{C}$ holding chamber. After $15 \mathrm{~min}$, slices were transferred to room-temperature artificial CSF (ACSF) containing the following (in mM): $\mathrm{NaCl}, 125 ; \mathrm{KCl}, 3.5 ; \mathrm{MgCl}_{2}, 1.3 ; \mathrm{NaH}_{2} \mathrm{PO}_{4}, 1.2$; $\mathrm{CaCl}_{2}$, 2.4; glucose, 25; $\mathrm{NaHCO}_{3}$, 26; gassed with $95 \% \mathrm{O}_{2}$ and $5 \% \mathrm{CO}_{2}$ ) until used for recordings over the subsequent $4 \mathrm{~h}$.

Nuclear calcium imaging in acute brain slices. Single slices were transferred to a recording chamber (OAC-1; Science Products), secured with a platinum harp, and submerged with continuously flowing $(3 \mathrm{ml} / \mathrm{min})$ ACSF maintained at $32-34^{\circ} \mathrm{C}$ with an in-line perfusion heater (TC324B; Warner Instruments). Wide-field imaging was performed using a fixed-stage upright microscope (BX51WI; Olympus), a $20 \times$ objective (XLUMPLFL20xW, numerical aperture 1.0; Olympus), an sCMOS (Andor Neo; Acal BFi) or EMCCD camera (Andor Ixon Ultra888; Visitron Systems), and a software interface (Andor iQ or Visiview; Visitron Systems). GCaMP3.NLS was imaged with 470/40 nm excitation and 525/50 emission filters (AHF Analysentechnik). Most imaging experiments were accompanied by whole-cell patch-clamp recordings from CA1 hippocampal pyramidal neurons (see below). Images were analyzed as 
A
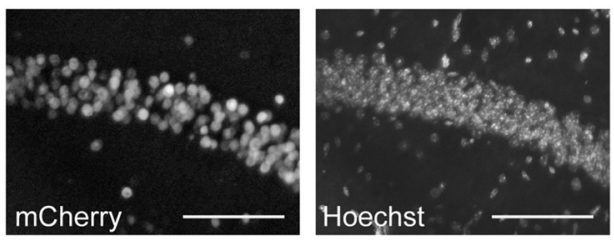

C

rAAV- $m C . N L S$

rAAV-PV.NLS- $m C$

rAAV- $m C . N L S+r A A V-V E G F D$

rAAV-PV.NLS- $m C+$ rAAV-VEGFD

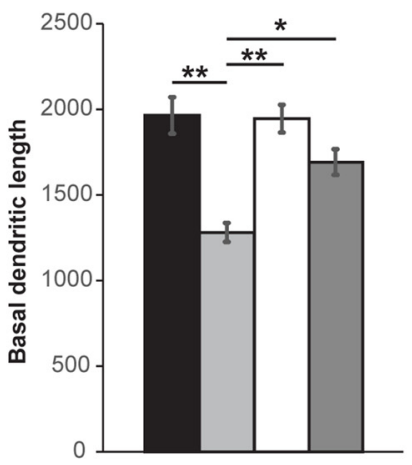

E

mC.NLS + hrGFP

hrGFP

PV.NLS-mC+ hrGFP

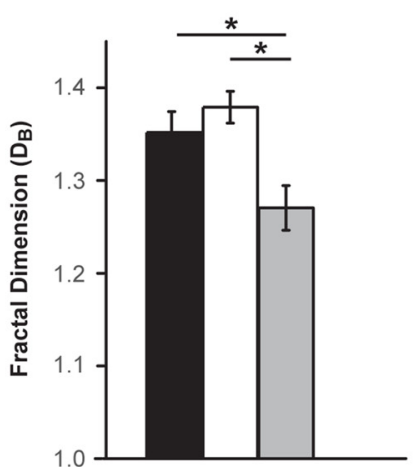

B

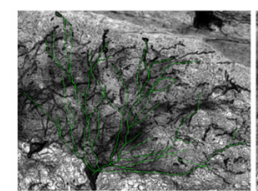

IAAV- $m$ C.NLS

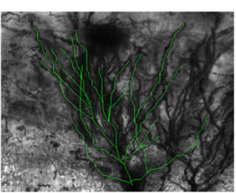

rAAV-PV.NLS- $m C$

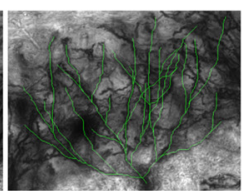

rAAV-PV.NLS- $m C$ + rAAV-VEGFD

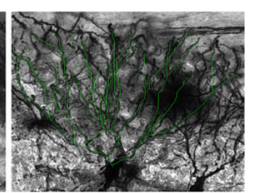

rAAV- $m C . N L S$

+ rAAV-VEGFD

D
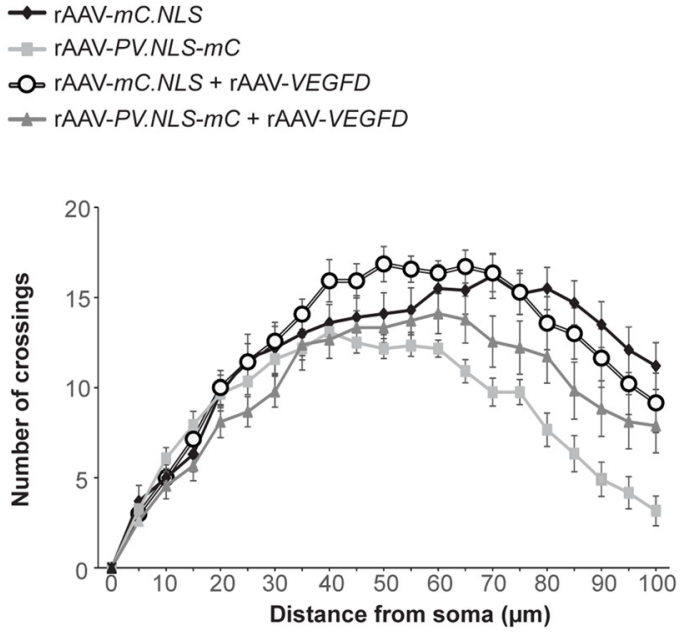

F

$\square$ rAAV- $m C . N L S$
$\square$ rAAV- $m C . N L S+$ rAAV-VEGFD
$\square$ rAAV-PV.NLS- $m C$
$\square$ rAAV-PV.NLS- $m C+$ rAAV-VEGFD

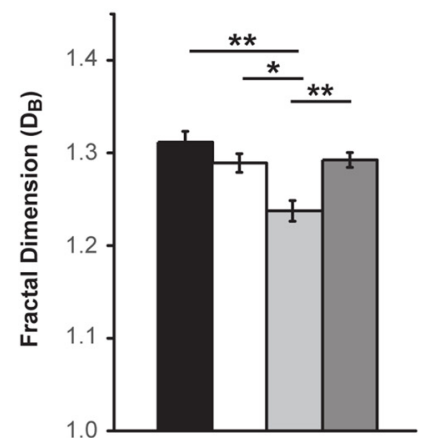

G

$\square$ rAAV-empty $m C$
$\square$ rAAV-shSCR
$\square$ rAAV-shVEGFD

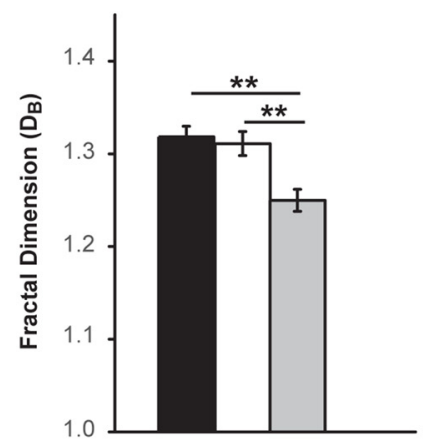

Figure 1. Generation of reduced-complexity dendritic arbors of adult mouse CA1 pyramidal neurons through blockade of nuclear calcium signaling and its rescue with VEGFD. $A$, Representative images of PV.NLS-mC expression in the CA1 region of the hippocampus of mice stereotaxically injected into the dorsal hippocampus with rAAV-PV.NLS-mC. MCherry fluorescence is shown (left); nuclei of cells were counterstained with Hoechst 33258 (right). Scale bar, $100 \mu \mathrm{m}$. B, Representative images and corresponding tracings (green lines) of the basal dendrites of Golgi-stained CA1 pyramidal neurons of mice stereotaxically injected into the dorsal hippocampus with either rAAV-mC.NLS, rAAV-PV.NLS-mC, rAAV-mC.NLS plus rAAV-VEGFD or rAAV-PV.NLS-mC plus rAAV-VEGFD. $C$, Analysis of the length of the basal dendrites of Golgi-stained CA1 pyramidal neurons of mice stereotaxically injected into the dorsal hippocampus with the indicated viruses. $r A A V-m C$.NLS, $n=10$ neurons from 5 animals; rAAV-PV.NLS- $m C, n=12$ neurons from 4 animals; rAAV- $m$ C.NLS plus rAAV-VEGFD, $n=14$ neurons from 4 animals; rAAV-PV.NLS- $m$ C plus rAAV-VEGFD, $n=18$ neurons from 4 animals $\left(F_{(3,50)}=13.848\right.$, rAAV- $m$ C.NLS vs rAAV-PV.NLS- $m$ C $p=0.00000147$; rAAV-PV.NLS- $m$ C plus rAAV-VEGFD vs rAAV-PV.NLS- $m$ C $p=0.003 ;$ rAAV- $m$ C.NLS plus rAAV-VEGFD vs rAAV-PV.NLS $p=0.000000798$, one-way ANOVA followed by Tukey's multiple-comparisons test). $\boldsymbol{D}$, Sholl analysis of the basal dendrites from $\boldsymbol{C}$. $\boldsymbol{E}$, Fractal dimension of the dendritic arbor of cultured mouse hippocampal neurons transfected with an expression vector for hrGFP $(n=10$ neurons from 4 independent cultures) or cotransfected with expression vectors for hrGFP and $\mathrm{mC}$.NLS $(n=12$ neurons from 4 independent cultures) or with expression vectors for hrGFP and PV.NLS-mC ( $n=11$ neurons from 4 independent cultures $\left(F_{(2,30)}=6.499\right.$, $\mathrm{mC}$.NLS vs PV.NLS-mC $p=0.031$, hrGFP vs PV.NLS-mC, $p=0.0049$, one-way ANOVA followed by Tukey's multiple-comparisons test). $\boldsymbol{F}$, Fractal dimensions of the basal dendrites of Golgi-stained CA1 pyramidal neurons of mice stereotaxically injected into the dorsal hippocampus with rAAV- $m$ C.NLS $(n=19$ neurons from 8 animals), rAAV- $m$ C.NLS plus rAAV-VEGFD ( $n=18$ neurons from 4 animals), rAAV-PV.NLS- $m C$ ( $n=25$ neurons from 8 animals), or rAAV-PV.NLS- $m$ C plus rAAV-VEGFD ( $n=13$ neurons from 4 animals $\left(F_{(3,71)}=10.198\right.$, rAAV- $m$ C.NLS vs rAAV-PV.NLS- $m C, p<0.00001$, rAAV-PV.NLS- $m$ C vs rAAV-PV.NLS- $m$ C plus rAAV-VEGFD $p=0.00184$, rAAV-PV.NLS-m ( vs rAAV-mC.NLS plus rAAV-VEGFD $p=0.01067$, one-way ANOVA followed by Tukey's multiple-comparisons test). G, Fractal dimension of the basal dendrites of Golgi-stained CA1 pyramidal neurons of mice stereotaxically injected into the hippocampus with rAAV-empty. $m C$ ( $n=19$ neurons from 4 mice), rAAV-shSCR ( $n=19$ neurons from 4 mice), or rAAV-shVEGFD ( $n=19$ neurons from 4 mice $\left(F_{(2,54)}=9.74\right.$, rAAV-empty.m ( vs rAAV-shVEGFD $p<0.00001$, rAAV-shSCR vs rAAV-shVEGFD $p=0.002$, one-way ANOVA followed by Tukey's multiple-comparisons test). Histograms indicate mean \pm SEM. 
A

rAAV- $m C . N L S$

rAAV-PV.NLS- $m C$

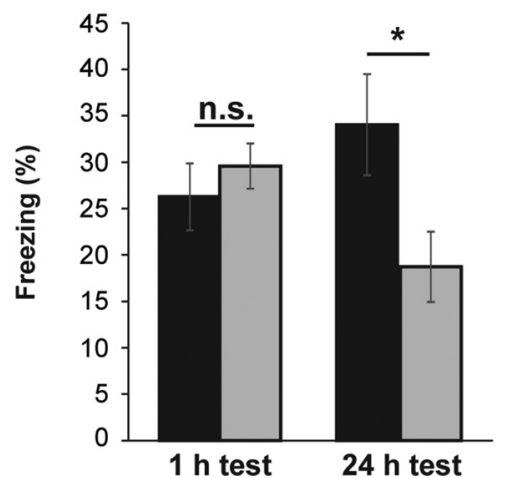

B

rAAV- $m C . N L S$

rAAV-PV.NLS- $m C$

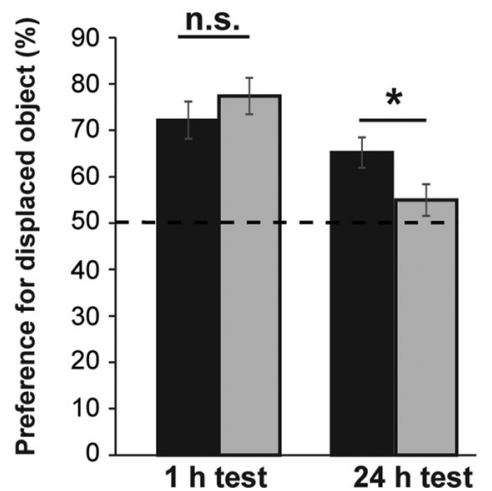

C

rAAV-mC.NLS

rAAV-PV.NLS- $m C$

rAAV-mC.NLS + rAAV-VEGFD

rAAV-PV.NLS-mC + rAAV-VEGFD

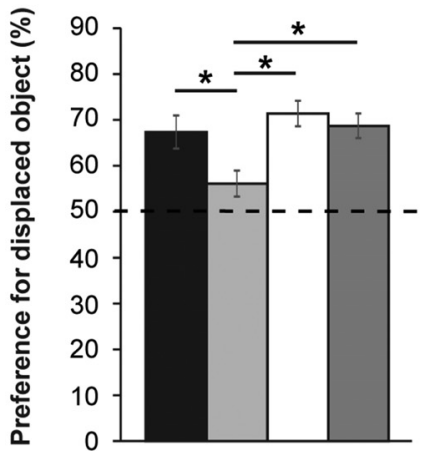

24 h test

Figure 2. Nuclear calcium-VEGFD signaling is important for long-term but not short-term memory. $A$, Short-term and long-term contextual fear memory of mice stereotaxically injected into the dorsal hippocampus with the indicated viruses. Results are expressed as the percentage of time mice spent freezing during the contextual memory test $1 \mathrm{~h}$ (short-term memory: rAAV-mC.NLS $n=$ 12, rAAV-PV.NLS- $m C n=12, t_{(22)}=0.757, p=0.457$, independent-samples $t$ test) or $24 \mathrm{~h}$ after training (long-term memory: rAAV- $m C . N L S n=10$, rAAV-PV.NLS- $m C n=9, t_{(17)}=2.317, p=$ 0.033 , independent samples $t$ test). $\boldsymbol{B}$, Short-term and long-term object-location memory of mice stereotaxically injected into the dorsal hippocampus with the indicated viruses. Animals were tested one hour (short-term memory: rAAV-mC.NLS $n=7$, rAAV-PV.NLS- $m C n=8, t_{(13)}=0.926, p=0.371$, independent-samples $t$ test) or $24 \mathrm{~h}$ after training (long-term memory rAAV- $m$ C.NLS $n=13$, rAAV-PV.NLS- $m C n=13, t_{(24)}=2.168, p=0.04$, independent-samples $t$ test). C, Long-term object-location memory of mice stereotaxically injected into the dorsal hippocampus with the indicated viruses. RAAV-mC.NLS, $n=16$; rAAV-PV.NLS- $m C, n=16$; rAAV-mC.NLS plus rAAV-VEGFD, $n=15 ;$ rAAV-PV.NLS-mC plus rAAV-VEGFD $n=16\left(F_{(3,59)}=5.028\right.$, rAAV-mC.NLS vs rAAV-PV.NLS- $m C, p=0.048$; rAAV-PV.NLS- $m$ C plus rAAV-VEGFD vs rAAV-PV.NLS, $p=0.021$; rAAV- $m$ C.NLS plus rAAV-VEGFD vs rAAV-PV.NLS, $p=0.004$, one-way ANOVA followed by Tukey's multiple-comparisons test). Histograms indicate mean \pm SEM; n.S., not significant.

$\Delta F / F_{0}=\left(F-F_{0}\right) / F_{0}$, where $F$ represents the average background subtracted emission fluorescence intensity in a region of interest (ROI) and $F_{0}$ represents the baseline $F$ measured before each stimulation. At the end of all recordings, depolarizing solution containing $45 \mathrm{~mm} \mathrm{~K}^{+}$and $4 \mathrm{~mm}$ $\mathrm{Ca}^{2+}$ was applied to the slice to elicit a near maximal calcium response. ROIs were assigned using images of both GCaMP3.NLS during depolarization and mCherry to ensure coexpression of both constructs in the cells analyzed.

Whole-cell patch-clamp electrophysiology. Patch electrodes (3-4 M $\Omega$ ) were made from $1.5 \mathrm{~mm}$ borosilicate glass and filled with internal solution containing the following (in $\mathrm{mM}$ ): potassium gluconate, $118 ; \mathrm{NaCl}$, 10; KCl, 20; HEPES, 10; EGTA, 0.2; $\mathrm{K}_{2}$-phosphocreatine, 10; $\mathrm{Mg}_{2} \mathrm{ATP}, 2$; $\mathrm{Na}_{3} \mathrm{GTP}, 0.3$; pH 7.35 with $\mathrm{KOH}$ ). Recordings were made with a Multiclamp 700A amplifier, digitized through a Digidata 1322A A/D converter and acquired and analyzed using pClamp 10 software (Molecular Devices). Access resistance (range: $10-20 \mathrm{M} \Omega$ ) was monitored regularly during voltage-clamp recordings and data were rejected if changes $>20 \%$ occurred. Some smoothing of EPSC traces was performed for display purposes. Membrane potentials have been corrected for a junction potential of $11 \mathrm{mV}$. Action potential (AP) threshold was determined from the time of the first positive peak in the third derivative of the spike waveform (Henze and Buzsáki, 2001). AP amplitude was determined relative to the AP threshold from an AP evoked by a minimal current injection for $1 \mathrm{~s}$. The accommodation index was calculated as the ratio of the time interval between the first and last pairs of APs in response to a $1 \mathrm{~s}$ current injection evoking an uninterrupted sequence of at least 6 APs. Postsynaptic currents were evoked using an ACSF-filled patch electrode positioned in the distal stratum radiatum and stratum oriens 210 and $100 \mu \mathrm{m}$ from stratum pyramidale, respectively, receiving $100 \mu \mathrm{s}$ stimuli from constant current stimulus isolator units (A365; World Precision Instruments) in bipolar mode (alternating polarity). Stimulation intensity was $100 \mu \mathrm{A}$ for all high-frequency stimulation (HFS) recordings. Stimulus artifacts have been removed from some traces.

Hippocampal neuronal cultures. Hippocampal neurons from newborn $\mathrm{C} 57 \mathrm{Bl} / 6$ mice were prepared as described previously (Bading and Greenberg, 1991), except that growth medium was supplemented with B27 (Invitrogen) and $1 \%$ rat serum (v/v). Cultures were infected with rAAVs at $4 \mathrm{~d}$ in vitro (DIV4). Experiments were conducted at DIV10. Bicuculline was used at a concentration of $50 \mu \mathrm{M}$ (Alexis Biochemicals).

$q R T-P C R$. The RNeasy Plus Mini Kit (Qiagen) was used for RNA isolation with additional on-column DNase I digestion according to the manufacturer's instructions. For cDNA synthesis, $1 \mu \mathrm{g}$ of total RNA was reverse transcribed with the High-Capacity cDNA reverse transcription kit (Invitrogen). qRT-PCR was performed on an ABI7300 thermal cycler using TaqMan gene expression assays (Applied Biosystems) for the following genes: Dnmt3a2 (Mm00463987_m1), BdnfIV (Mm00432069_m1), Nr4a1 (Mm00439358_m1), Npas4 (Mm00463644_m1), and Egr1 (Mm00656724_m1). Expression levels of target genes were normalized to the expression of the housekeeping gene Gusb (Mm00446953_m1). For each independent experiment, the fold induction of the uninfected control upon bicuculline stimulation was set to $100 \%$ and remaining conditions were normalized accordingly.

Experimental design and statistical analysis. The behavioral data were collected from two to three sets of experiments. Each set of experiments contained mice injected with control or experimental viruses and were randomized per cage (i.e., each cage of four mice contained mice injected with control or experimental viruses). Experiments were performed 3 weeks after rAAV injection except for the extinction experiment (see Fig. $3 A$ for timeline). In vivo experiments were done at least in duplicate and included four to five mice (10-25 neurons) for the morphometric analysis and 8-16 mice for behavioral experiments. Acute brain slice experiments were performed in five to 10 mice (20-42 slices) per group for imaging and four to 10 mice (four to 21 neurons) per group for electrophysiological analyses as indicated in the table and figure legends for each experiment. Cell culture experiments were performed from four independent cultures. For behavioral, imaging, electrophysiological, and morphometric studies, the experimenter was blind to treatment until analysis was performed. All plotted data represent mean \pm SEM unless otherwise stated. The Shapiro-Wilk test was used to test for normality. Independent-samples $t$ tests were used to compare two distinct groups. For comparison of more than two groups, one-way or two-way ANOVA followed by Tukey's post hoc tests were applied. For the nuclear calcium imaging results, statistical comparisons were performed on rank-ordered data due to the non-normal distribution of the raw data. A mixed-design 
A

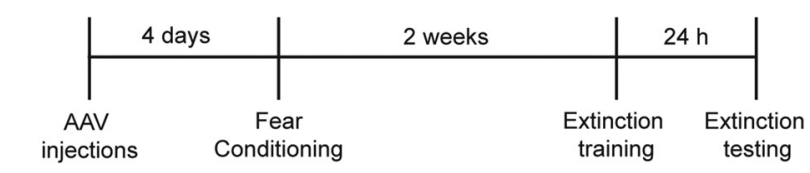

C

rAAV-mC.NLS

RAAV-PV.NLS- $m C$

rAAV-mC.NLS + rAAV-VEGFD

rAAV-PV.NLS- $m C+$ rAAV-VEGFD

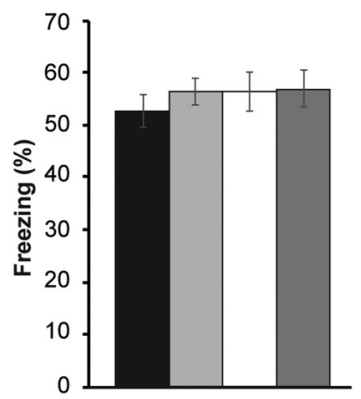

Ext 1

\section{D}

rAAV- $m C . N L S+$ rAAV-VEGFD
rAAV-PV.NLS- $m C+$ rAAV-VEGFD

$E$

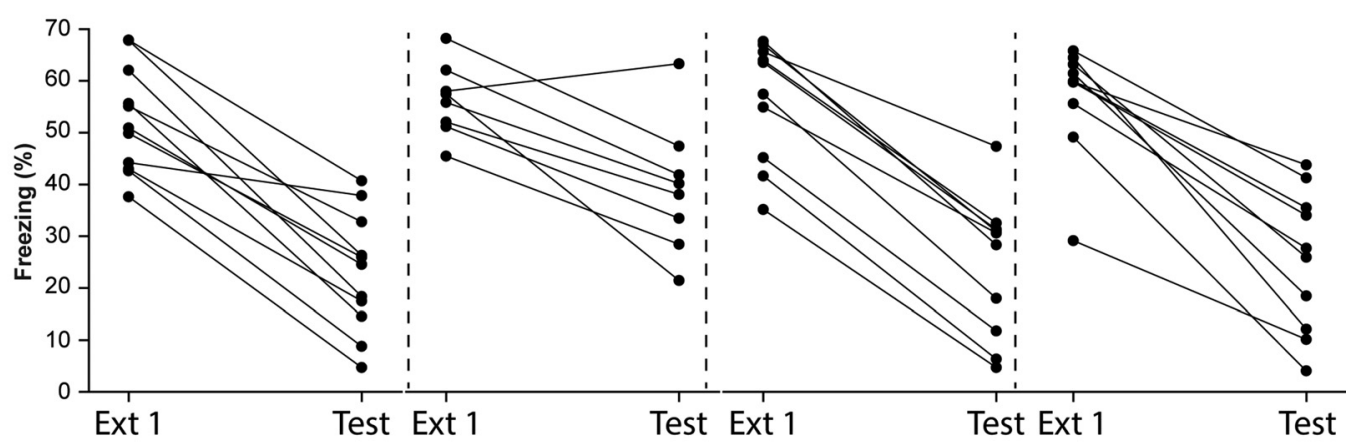

\section{$\sim$ rAAV-mC.NLS}

- rAAV-PV.NLS-mC

$=0$ rAAV- $m C . N L S+$ rAAV-VEGFD

$\tau$ rAAV-PV.NLS- $m C+$ rAAV-VEGFD

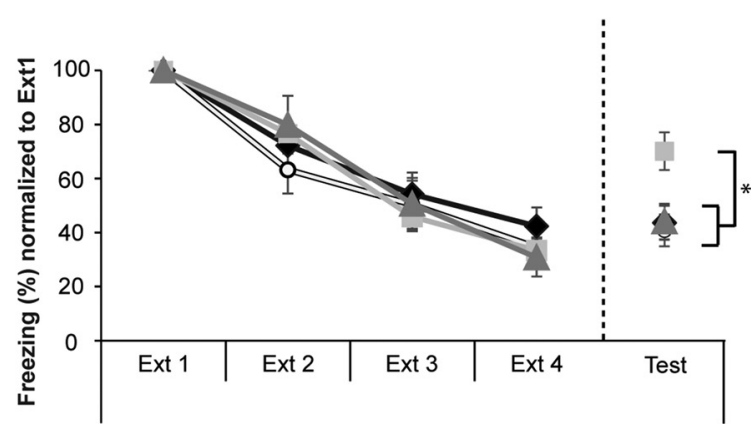

Figure 3. Dendritic morphology is critical for fear memory extinction. $\boldsymbol{A}$, Schematic illustration of the experimental timeline. $\boldsymbol{B}$, Representative pictures of virus-mediated mCherry expression $4 \mathrm{~d}$ and 3 weeks after stereotaxic delivery. Scale bar, $100 \mu \mathrm{m}$. C, Freezing responses exhibited during the first 5 min of the extinction training phase by mice stereotaxically injected into the dorsal hippocampus with the indicated viruses. RAAV- $m$ C.NLS, $n=11$; rAAV-PV.NLS- $m C, n=8$; rAAV- $m$ C.NLS plus rAAV-VEGFD, $n=10 ;$ rAAV-PV.NLS- $m$ C plus rAAV-VEGFD, $n=10$ ( $F_{(3,35)}=0.415$, rAAV- $m$ C.NLS vs rAAV-PV.NLS- $m$ C $p=0.8475$; rAAV-PV.NLS- $m$ C plus rAAV-VEGFD vs rAAV-PV.NLS- $m$ C, $p=0.9995$; rAAV- $m$ C.NLS plus rAAV-VEGFD vs rAAV-PV.NLS, $p>0.9999$, one-way ANOVA followed by Tukey's multiple-comparisons test). $D$, Pointing to dot representation showing raw data of freezing responses for each individual animal from Ext1 and Test session to indicate within group variability. $\boldsymbol{E}$, Results are expressed as the percentage of time mice spent freezing in relation to the first 5 min of the extinction training phase. The freezing responses during the extinction training phase (Ext $1-4)$ and test session $24 \mathrm{~h}$ after (Test) are plotted $\left(F_{(3,35)}=3.986\right.$, rAAV- $m$ C.NLS vs rAAV-PV.NLS- $m C, p=0.0352 ;$ rAAV-PV.NLS- $m$ C plus rAAV-VEGFD vs rAAV-PV.NLS- $m$ C, $p=$ 0.0449; rAAV-m C.NLS plus rAAV-VEGFD vs rAAV-PV.NLS, $p=0.0196$, one-way ANOVA followed by Tukey's multiple-comparisons test). Ext $1-4$ indicate 5 min time blocks of extinction training. Data in $\boldsymbol{A}$ and $\boldsymbol{C}$ indicate mean \pm SEM.

two-way ANOVA was used with six HFS repetitions as the repeatedmeasures factor and the sh construct (shSCR versus shVEGFD) as the between-groups factor. Prism7 and OriginPro 2016 were used for statistical analysis.

\section{Results}

To interfere with nuclear calcium-VEGFD signaling in the adult mouse hippocampus in vivo, we delivered stereotaxically into the mouse hippocampus an rAAV containing an expression cassette for the calcium binding protein parvalbumin (PV) fused to a nuclear localization signal and mCherry (rAAV-PV.NLS-mC) (Mauceri et al., 2015) (Fig. 1A). In cultured hippocampal neurons, expression of PV.NLS-mC increases nuclear calcium buffering, leading to a reduction of the dendrite length and complexity. This is caused by a decreased expression of the nuclear calcium target gene VEGFD (Mauceri et al., 2015). We first assessed the effect of expression of PV.NLS-mC on the morphology of CA1 Golgi-stained pyramidal neurons using Sholl analysis. Compared with control neurons expressing mCherry.NLS (rAAV- 

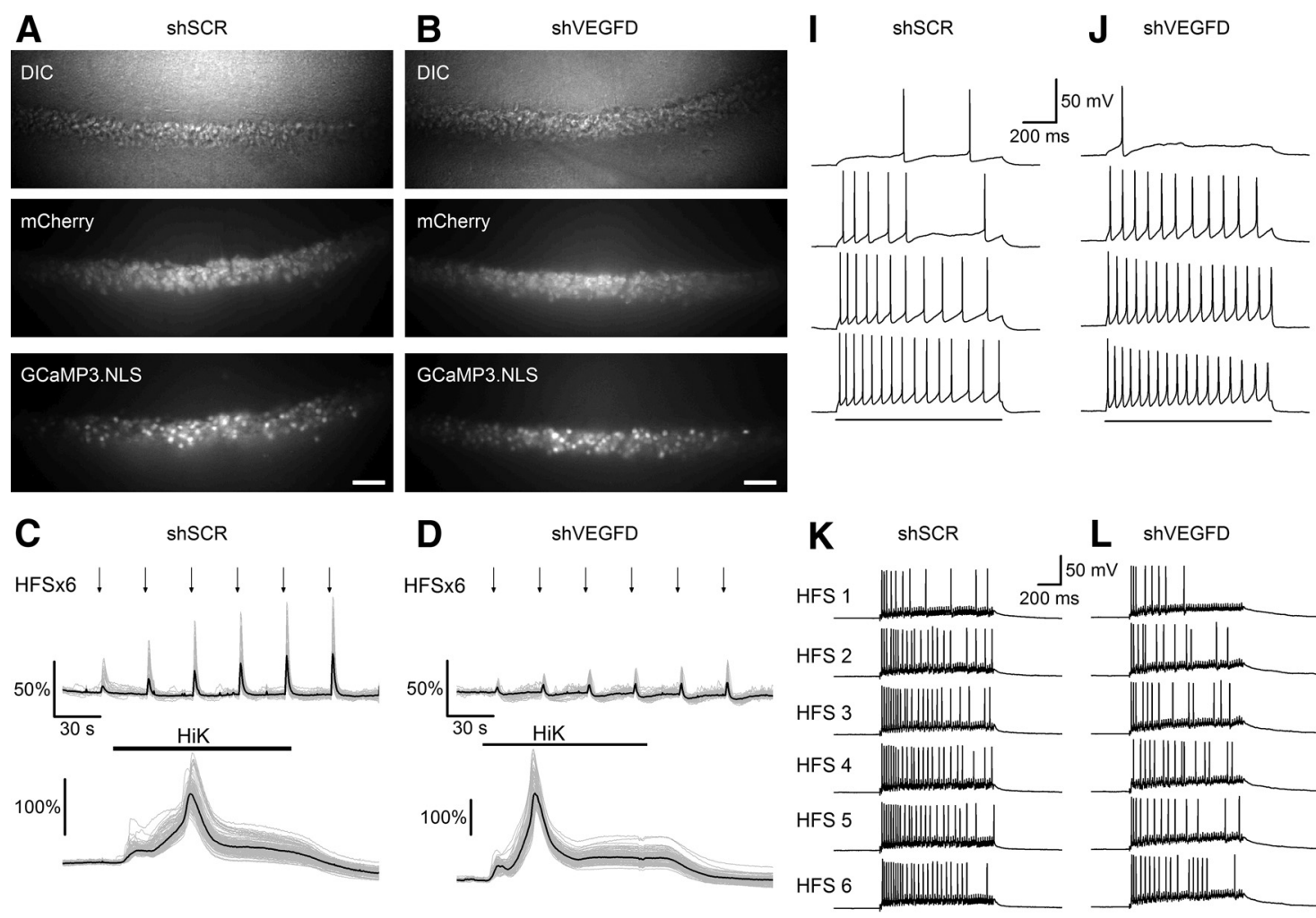

L ShVEGFD
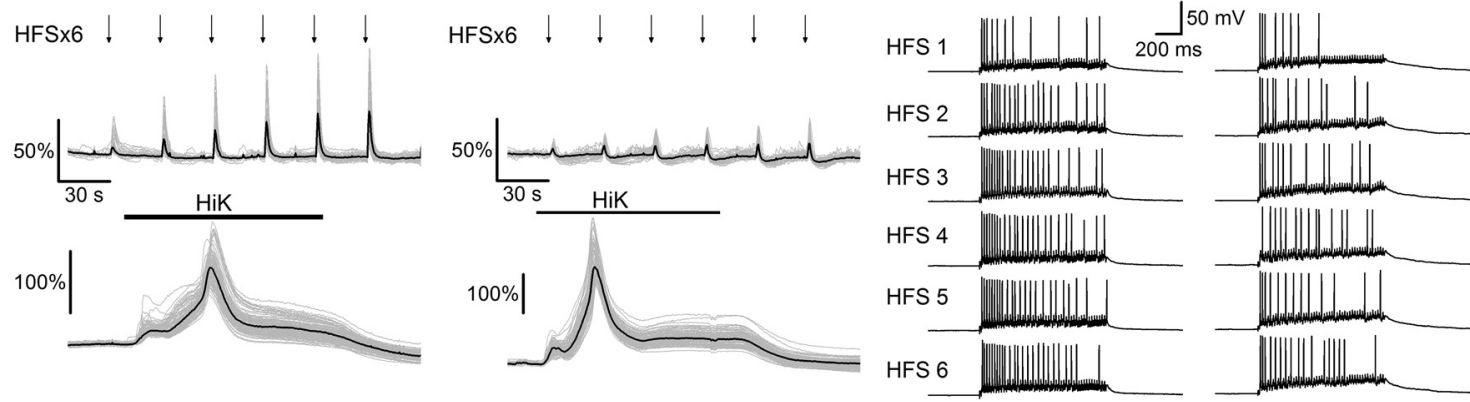

E
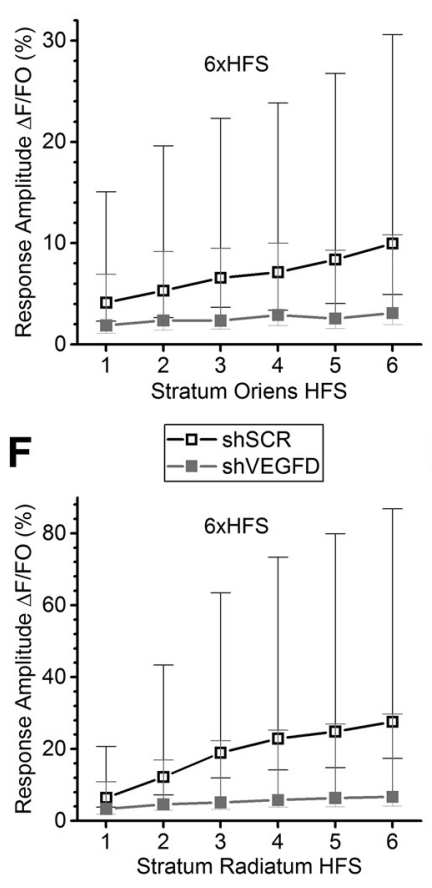

G

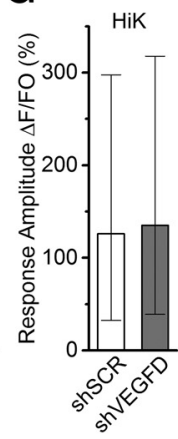

H

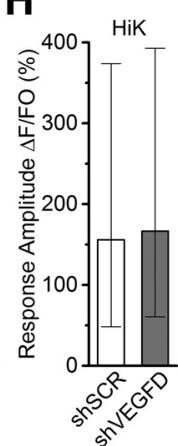

M

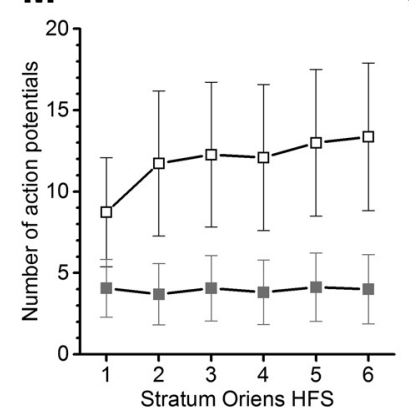

0

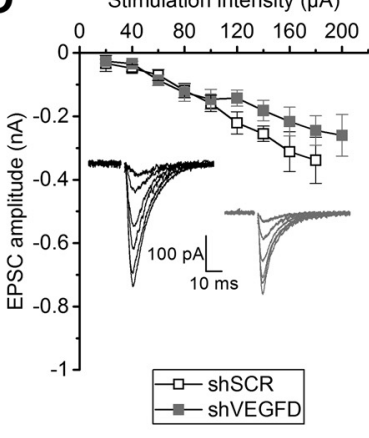

N

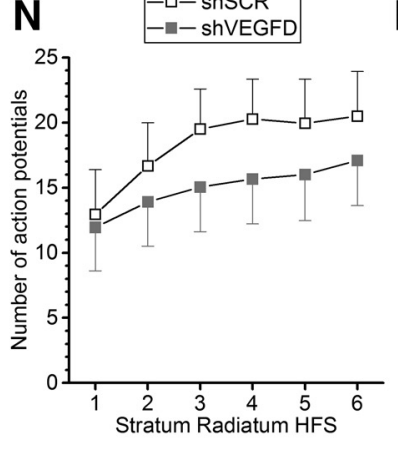

P

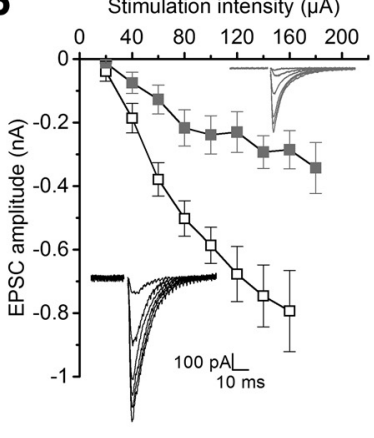

Figure 4. A compromised dendritic morphology impairs synapse to nucleus calcium signaling. $A, B$, Images showing differential interference contrast (DIC), $m C$ herry, and GCaMP3.NLS of neurons coexpressing GCaMP3.NLS and either shSCR.mCherry $(\boldsymbol{A})$ or shVEGFD.mCherry $(\boldsymbol{B})$ in the CA1 stratum pyramidale. Scale bar, $50 \mu \mathrm{m}$. $\boldsymbol{C}, \boldsymbol{D}$, Representative slice recordings of nuclear calcium responses to 6 high-frequency stimulations (HFS1-6, $100 \mu \mathrm{A}, 100 \mathrm{~Hz}, 1 \mathrm{~s}$ repeated every $30 \mathrm{~s}$ ) of Schaffer collateral fibers in the stratum radiatum. Traces below show the responses of the same cells to depolarizing HiK. Gray traces represent individual cells and black traces their average. $\boldsymbol{E}-\boldsymbol{H}$, Nuclear calcium responses to HFS $(\boldsymbol{E}, \boldsymbol{F})$ and HiK $(\boldsymbol{G}, \boldsymbol{H})$ in experiments with the stimulator in either the stratum oriens $(\boldsymbol{E}, \boldsymbol{G})$ or radiatum $(\boldsymbol{F}, \boldsymbol{H})$. Graphs show the median and interquartile range of the peak response amplitudes quantified from the $\Delta F / F_{0}$ of the 50 most responsive cells per slice. There was a significant effect of virus group on HFS responses ( 2 way ANOVA; oriens $F_{(1,1998)}=376, p<0.00001,1000$ cells in 20 slices from 5 mice per group; radiatum: $F_{(1,4198)}=722, p<0.00001$, 2100 cells in 42 slices from 10 mice per group) and a smaller effect on HiK responses in the oriens cell group (1 way ANOVA $\left.F_{(1,1998)}=9.21, p=0.0024\right)$, but not the radiatum cell group $\left(F_{(1,4198)}=\right.$ $2.01, p=0.156$ ). $\boldsymbol{I}-\boldsymbol{L} \boldsymbol{L}$, Traces showing examples of whole-cell patch-clamp recordings of APs generated in response to current injection (black line, 20 to $80 \mathrm{pA}$ in $20 \mathrm{pA}$ increments; $\boldsymbol{I}, \boldsymbol{J}$ ) and six repetitions of HFS in the stratum radiatum $(\boldsymbol{K}, \boldsymbol{L})$ in shSCR- and shVEGFD-expressing neurons. $\boldsymbol{M}, \boldsymbol{N}$, Mean ( \pm SEM) numbers of APs generated in response to HFS of stratum oriens $(\boldsymbol{M})$ or radiatum $(\boldsymbol{N})$. HFS-evoked spike numbers were highly variable between cells and total spike numbers were not significantly different between the sh construct groups (stratum radiatum: independentsamples $t$ test $t_{(37)}=0.716, p=0.479$, shSCR, $n=18$; shVEGFD, $n=21$; stratum oriens: $t_{(27)}=1.867, p=0.073$; shSCR, $n=13$; shVEGFD, $n=16$ ). (Figure legend continues.) 
$m C . N L S)$, neurons expressing PV.NLS-mC showed a reduction in both the complexity and total length of their basal dendrites (Fig. $1 B-D$ ). The expression of VEGFD, by means of a stereotaxically injected rAAV containing an expression cassette for HAtagged VEGFD (rAAV-VEGFD), almost completely restored dendrite length and Sholl-analysis-based complexity (Fig. 1B-D). For a comparative analysis of dendrite geometries, it is instructive to go beyond the standard assessment of neuronal morphology. We therefore analyzed the fractal dimension $D_{\mathrm{B}}$, which accurately reflects the complexity of form and the space-filling properties of an object, allowing the description of the geometrical feature of a dendritic arbor with a single index (Smith et al., 1996; Fernández and Jelinek, 2001; Puškaš et al., 2015; see Materials and Methods). Analysis of control neurons (expressing either hrGFP or hrGFP plus mCherry.NLS) and reduced-complexity neurons (expressing PV.NLS-mC plus hrGFP) in dissociated cultures of hippocampal neurons revealed significant differences in their $D_{\mathrm{B}}$ (Fig. 1E; images from Mauceri et al., 2015). In Golgi-stained CA1 pyramidal neurons of the mouse brain (Fig. $1 B-D$ ), we detected a robust reduction in $D_{\mathrm{B}}$ for CA1 neurons expressing PV.NLS-mC compared with neurons expressing mCherry.NLS, which was fully rescued by VEGFD (Fig. $1 F$ ). Conversely, knock-down of VEGFD using shVEGFD in vivo, which we have shown previously to reduce dendrite length and complexity and to impair memory consolidation (Mauceri et al., 2011), yielded values for $D_{\mathrm{B}}$ that were virtually identical to that obtained after nuclear calcium buffering with PV.NLS (Fig. $1 G$ ).

Having established a means of fine tuning dendritic architecture of CA1 neurons in vivo, we next investigated its impact on cognitive abilities. Using hippocampus-dependent memory tests, we found that structural alterations induced by expression of PV.NLS-mC generates a behavioral phenotype similar to that shown previously for VEGFD knock-down (Mauceri et al., 2011). Mice with reduced complexity of their CA1 basal dendrites due to expression of PV.NLS-mC lost their ability to form hippocampus-dependent long-term memory in both CFC and SOR tests (Fig. 2A,B). Formation of short-term memories was not compromised by the loss of dendritic structures caused by PV.NLS-mC. Moreover, long-term deficits in SOR caused by PV.NLS-mC expression were fully rescued by restoring dendrite complexity with VEGFD (Fig. 2C). Therefore, interfering with nuclear calcium-VEGFD signaling with either PV.NLS-mC or shVEGFD-mC induces a compromised dendritic structure in vivo that is associated with long-term memory dysfunction.

Next, we investigated the impact of an altered dendritic architecture of CA1 neurons on fear memory extinction. Because PV.NLS-mC expression interferes with the consolidation of fear memories (see Fig. 2A), it was necessary to deliver the rAAVs for the extinction experiments at a time point ( $4 \mathrm{~d}$ before fear conditioning training; Fig. $3 A$ ) when the virus-mediated expression is too low to interfere with fear memory consolidation (Fig. $3 B$ ). This was confirmed by identical freezing response levels during the first 5 min of the extinction session in the PV.NLS-mC ex-

$\leftarrow$

(Figure legend continued.) $\quad \mathbf{O}, \boldsymbol{P}$, Synaptic input/output curves showing the relationship between field stimulation intensity and single EPSC amplitude recorded at $-70 \mathrm{mV}$ in the presence of gabazine $(5 \mu \mathrm{m})$ for stimulation applied in the stratum oriens $(\mathbf{0})$ or radiatum $(\boldsymbol{P})$. Mean \pm SEM are shown. Slopes estimated from linear fits of the curves were significantly different for stimulation of the stratum radiatum (independent-samples $t$ test $t_{(7)}=5.423 p=$ 0.001 , shSCR, $n=4$; shVEGFD, $n=5)$ but not stratum oriens $\left(t_{(15)}=0.656, p=0.521\right.$, shSCR, $n=6$; shVEGFD, $n=11$ ). Insets show example traces of responses to 40 to $140 \mu \mathrm{A}$ in $20 \mu \mathrm{A}$ increments from shSCR (black)- and shVEGFD (gray)-expressing cells.
Table 1. Effect of VEGFD knock-down on the electrophysiological properties of CA1 pyramidal neurons in acute slices

\begin{tabular}{lcc}
\hline & shSCR & shVEGFD \\
\hline Membrane capacitance (pS) & $84 \pm 4(45)$ & $80 \pm 4(48)$ \\
$V_{\text {rest }}(\mathrm{mV})$ & $-71.1 \pm 0.8(47)$ & $-72.0 \pm 0.8(46)$ \\
AP threshold (mV) & $-54.2 \pm 0.8(38)$ & $-48.8 \pm 1.0(37)^{*}$ \\
AP amplitude (mV) & $72.9 \pm 2.4(38)$ & $68.4 \pm 1.9(37)$ \\
AP half width (ms) & $1.30 \pm 0.04(40)$ & $1.40 \pm 0.08(40)$ \\
Accommodation index (AU) & $3.39 \pm 0.33(40)$ & $2.93 \pm 0.33(40)$
\end{tabular}

Parameters were quantified from whole-cell patch-clamp recordings in neurons infected with either rAAV-shSCR or rAAV-shVEGFD. Data represent mean \pm SEM (cell numbers are shown in parentheses).

$V_{\text {rest }}$, resting membrane potential.

*Independent-samples $t$ test: $t_{(73)}=4.343, p=0.000045$.

pressing mice and the control mice expressing mCherry.NLS (Fig. 3C). Both groups showed reduction of the fear-associated freezing behavior in the extinction training session (Fig. 3E, Ext14); in the test session $24 \mathrm{~h}$ later, mice expressing PV.NLS-mC showed higher levels of freezing than the control animals expressing mCherry.NLS (Fig. 3D,E). This result reflects an impairment in fear memory extinction. The ability to extinguish fear memory could be rescued by restoring nuclear calcium-VEGFD signaling and dendrite complexity using VEGFD expression (Fig. $3 D, E$ ).

The consolidation of extinction memory is a transcriptiondependent process (Vianna et al., 2001). Therefore, we considered the possibility that malfunction of the excitation-transcription coupling underlies the loss of extinction consolidation in mice with altered CA1 neuron dendrite geometry and investigated the generation of nuclear calcium transients. Because the nuclear calcium buffer PV.NLS reduces nuclear calcium transients per se (Mauceri et al., 2015), we generated reduced-complexity CA1 neurons in vivo via expression of shVEGFD. We measured synaptically evoked nuclear calcium signals in CA1 neurons of acute slices obtained from mice stereotaxically injected into the dorsal CA1 with an rAAV containing an expression cassette for the nuclear targeted recombinant calcium indicator GCaMP3.NLS (Bengtson et al., 2010; Weislogel et al., 2013) alongside rAAVs expressing either shVEGFD-mC or shSCR-mC (control) (Fig. 4A,B). Repeated trains of HFS of Schaffer collaterals in acute hippocampal slices induce robust nuclear calcium responses in the stratum pyramidale of CA1 (Bengtson et al., 2010) (Fig. 4C,D, top). Nuclear calcium responses to six repetitions of HFS in the stratum oriens or the stratum radiatum were dramatically reduced in shVEGFD$\mathrm{mC}$-expressing neurons compared with shSCR-mC neurons (Fig. 4E,F). To control for equivalent GCaMP3.NLS expression and calcium responsiveness between our treatment groups, we also measured responses to a depolarizing high potassium solution (HiK; Fig. 4C,D, bottom). Nuclear calcium responses to $\mathrm{HiK}$ were slightly increased in the shVEGFD dataset used for stratum oriens stimulation (Fig. 4G), but not in that used for stratum radiatum stimulation (Fig. $4 H$ ). Electrophysiological recordings in other slices showed no differences between shSCR- and shVEGFD-expressing cells in terms of passive properties of CA1 pyramidal neurons (membrane capacitance, resting membrane potential; Table 1). shVEGFD-expressing neurons showed a more depolarized threshold for AP initiation, although no difference in AP amplitude or half-width was evident and accommodation did not differ between the groups (Fig. 4I,J, Table 1). HFS-induced AP generation is a critical determinant of nuclear calcium response amplitude showing a parallel augmentation with HFS repetition (Bengtson et al., 2010). Stratum oriens- but not radiatum HFS-induced AP numbers showed a nonsignificant trend toward reduction in shVEGFD-expressing neurons (Fig. $4 K-N$ ). 
Uninfected

$\square$ rAAV-shSCR

$\square$ rAAV-shVEGFD
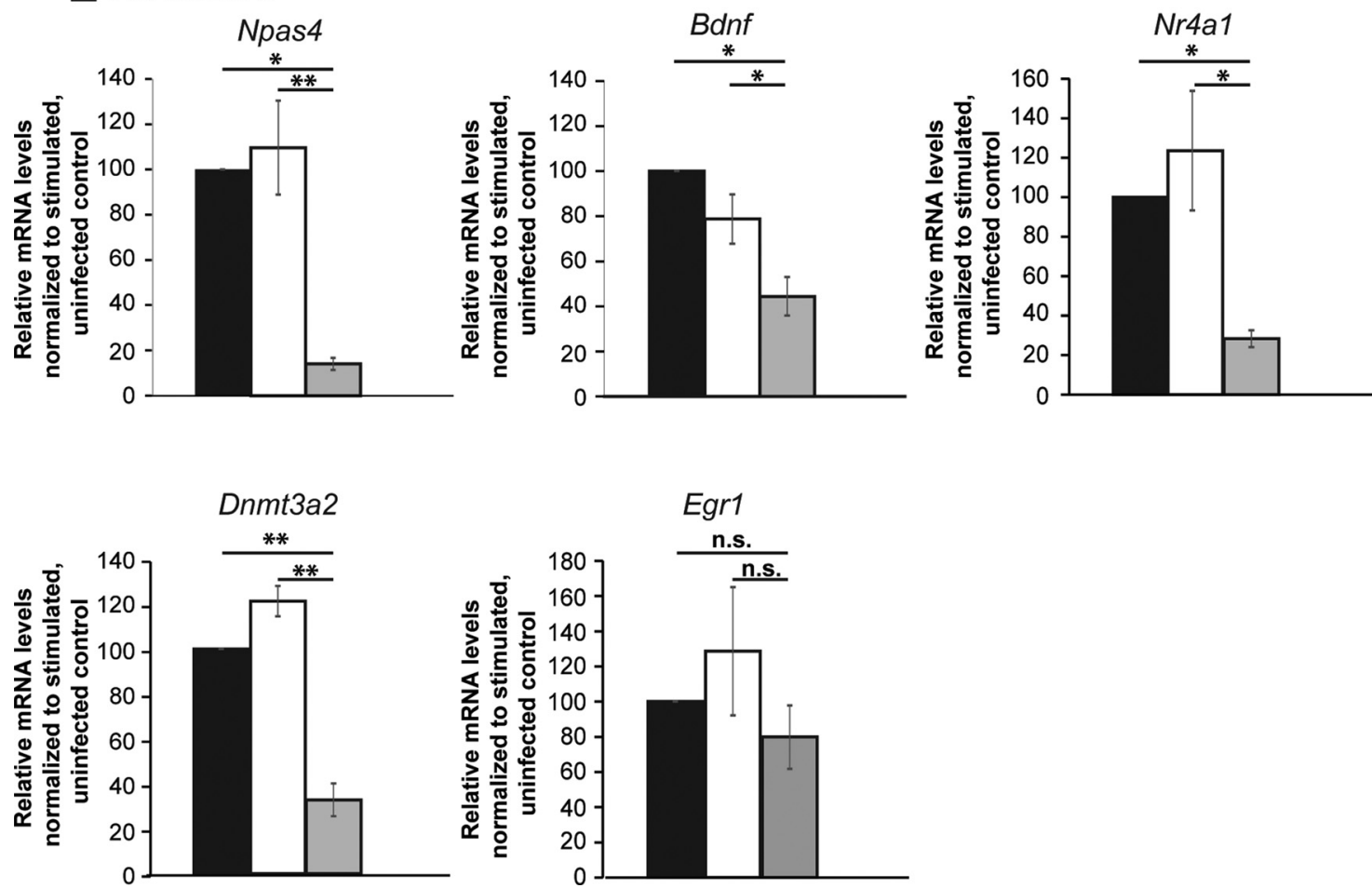

Figure 5. A compromised dendritic morphology impairs activity-dependent expression of plasticity-related genes. qRT-PCR analysis of expression of Npas4, Bdnf, Nr4a1, Dntm3a2, and Egr1 in uninfected hippocampal neurons $(n=4)$ and in hippocampal neurons infected with rAAV-shSCR $(n=4)$ or rAAV-shVEGFD $(n=4) 4 \mathrm{~h}$ after bicuculline stimulation (Npas4: $F_{(2,9)}=19.078$, uninfected vs rAAV-shVEGFD, $p=0.002$, rAAV-shSCR vs rAAV-shVEGFD, $p=0.001$ Bdnf: $F_{(2,9)}=12.261$, uninfected vs rAAV-shVEGFD, $p=0.002$, rAAV-shSCR vs rAAV-shVEGFD, $p=0.035 ;$;r4a1: $F_{(2.9)}=7.940$, uninfected vs rAAV-shVEGFD, $p=0.044$, rAAV-shSCR vs rAAV-shVEGFD, $p=0.01$; Dnmt3a2: $F_{(2.9)}=65.059$, uninfected vs rAAV-shVEGFD $p=0.000044$, rAAV-shSCR vs rAAV-shVEGFD, $p=0.000005 ;$ Egr1: $\left(F_{(2,9)}=1.094\right.$, uninfected vs rAAV-shVEGFD, $p=0.819$, rAAV-shSCR vs rAAV-shVEGFD, $p=0.348$, one-way ANOVA followed by Tukey's multiple-comparisons test). Histograms indicate mean $\pm \mathrm{SEM}$.

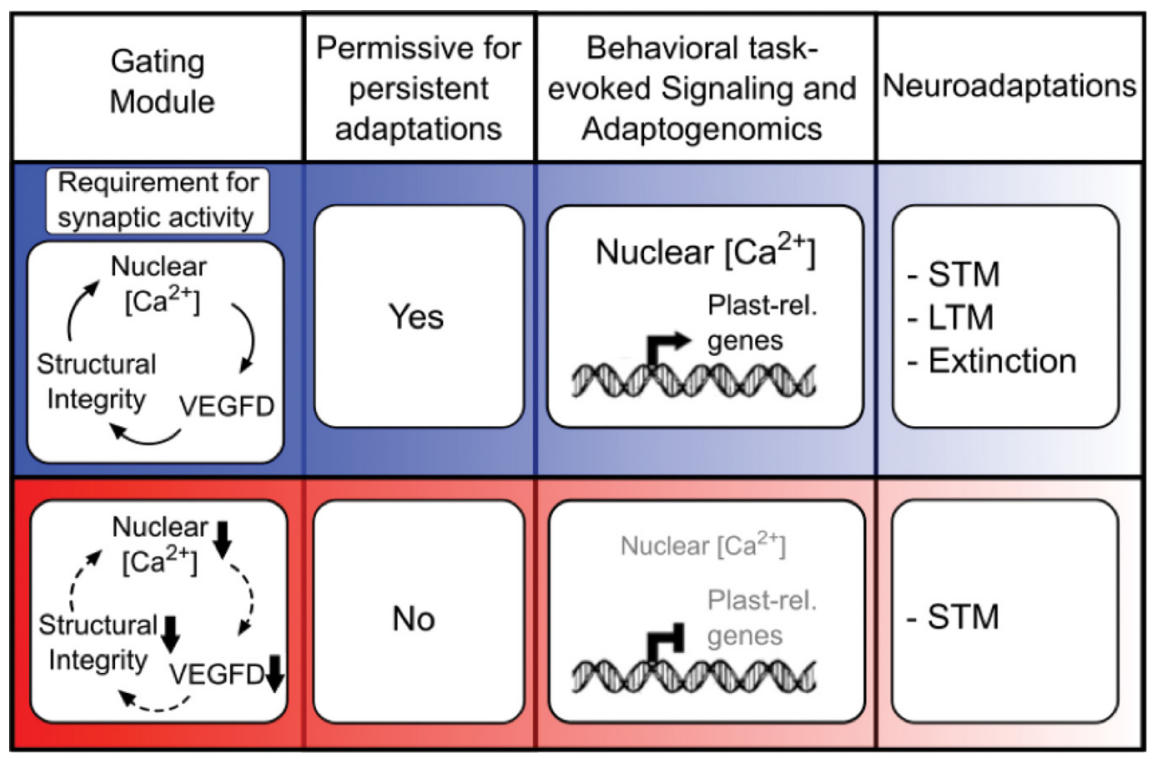

Figure 6. Schematic illustration of the "gating module" function in neuroadaptations. Disruption of any component of the gating module (i.e., nuclear calcium, VEGFD, structural integrity) negatively affects the other components and renders neurons nonpermissive for undergoing persistent neuronal adaptations. Malfunctional gating weakens the synapse-to-nucleus communication axis and impairs nuclear calcium-driven, plasticity-related (plast-rel.) gene expression required for long-term memory (LTM) and extinction, but not for short-term memory (STM).
To assess directly the effects of VEGFD knock-down, we measured the input/output function of AMPA-receptor-mediated EPSCs and found no apparent difference for stratum oriens stimulation but a reduced EPSC amplitude for stratum radiatum stimulation (Fig. 4O,P). The much larger EPSC amplitudes seen with highintensity stimulation in stratum radiatum compared with stratum oriens are likely due to the much larger dendritic volume of apical dendrites, which is activated incrementally by the larger electrical field of stronger stimulation pulses. A potential between-groups difference in the amplitude of EPSC responses to stratum oriens stimulation could not be further examined at higher stimulation intensities due to the emergence of nonsynaptic direct excitation. These results indicate that a reduction in CA1 dendrite complexity caused by VEGFD knock-down reduces excitatory synaptic afferent input to the apical dendrite and seriously impairs nuclear calcium transients evoked by bursts of synaptic activity at either apical or basal 
dendrites of CA1 pyramidal neurons. An shVEGFD-induced reduction in synaptic input is consistent with previous results showing a reduction in dendrite length and complexity without a change in spine density indicative of a reduced total synapse number after VEGFD knock-down (Mauceri et al., 2011).

This impairment in synapse-to-nucleus signaling most likely affects synaptic activity regulated transcription, which we investigated next. We infected primary hippocampal neurons with rAAV-shVEGFD or control virus and induced AP bursting known to induce robust nuclear calcium signals and a broad transcriptional response (Zhang et al., 2009; Bading, 2013) (Fig. 5). Compared with uninfected neurons or rAAV-shSCR infected neurons, neurons that expressed shVEGFD showed a reduction in activity-dependent expression of the plasticity-related genes Npas4, Nr4a1, Dnmt3a2, and Bdnf (Fig. 5), which are known to be important for memory formation and extinction (von Hertzen and Giese, 2005; Heldt et al., 2007; Ramamoorthi et al., 2011; Hawk et al., 2012; Oliveira et al., 2012, 2016). Activity-induced expression of Egrl, which is not dependent on nuclear calcium signaling (Zhang et al., 2009), but instead is controlled by several copies of the cytoplasmic calcium response element SRE (serum response element; Hardingham et al., 1997) in the Egrl promoter (Christy and Nathans, 1989), was not affected in cells expressing shVEGFD (Fig. 5). These results indicate that the structural integrity of neurons is necessary for a fully functional synapse-to-nucleus communication axis. Impairments of the dendrite geometry compromises nuclear calcium signaling, leading to a disruption of nuclear calciumdriven adaptogenomics that is needed for the consolidation process of both memory and extinction. Nuclear calcium signaling, VEGFD expression, and structural integrity are highly interdependent (Fig. 6) and thus form a "gating module" for neuroadaptations.

\section{Discussion}

In this study, we uncovered a mechanism responsible for structural integrity of CAl pyramidal neurons in the mouse hippocampus that ensures operational integrity of synapse-to-nucleus communication and nuclear calcium-driven transcription. Disruption of the reciprocally reinforcing interactions of dendrite geometry, nuclear calcium signaling, and gene regulation renders mice incapable of consolidating memory formation and extinction while sparing short-lasting neuroadaptations. Although reduced nuclear calcium responses caused by alterations in dendrite geometry effect a complex transcriptional response, supplementation of VEGFD helps to maintain structural integrity and cognitive functions in conditions of reduced nuclear calcium signaling.

Alterations in the hippocampal dendritic arbor are reported in stress-related anxiety disorders (Kassem et al., 2013; Cohen et al., 2014), causing impairments in the ability to extinguish aversive conditioned responses in both patients and animal models (Yehuda and LeDoux, 2007). This compromises the efficacy of extinction-based exposure therapies. Indeed, the return of anxiety-related responses to traumatic memories is a central feature of PTSD relapse even after therapy (Yehuda and LeDoux, 2007). Our findings suggest that a stress-dependent reduction of dendritic complexity may render the neuronal network less permissive to long-term persistence of new memories such as those acquired during extinction-based exposure therapies. Therefore, treatments aimed at stabilizing or even recovering the dendritic structure with VEGFD or other appropriate mimetics before the extinction-based behavioral therapy may boost their efficacy through the restoration of synapse-to-nucleus communication. Finally, neurodegenerative disorders, in particular those involving increased extrasynaptic NMDA signaling, are associated with distorted neuronal architecture, loss of synaptic connectivity, and deficits in gene regulation (Bading, 2017). Structure-protective treatments are expected to counteract the progressive loss of excitation-transcription coupling, thereby facilitating the expression of gene programs for neuroprotection and cognitive functions (West and Greenberg, 2011; Bading, 2013).

\section{References}

Agis-Balboa RC, Arcos-Diaz D, Wittnam J, Govindarajan N, Blom K, Burkhardt S, Haladyniak U, Agbemenyah HY, Zovoilis A, Salinas-Riester G, Opitz L, Sananbenesi F, Fischer A (2011) A hippocampal insulin-growth factor 2 pathway regulates the extinction of fear memories. EMBO J 30:40714083. CrossRef Medline

Bading H (2013) Nuclear calcium signalling in the regulation of brain function. Nat Rev Neurosci 14:593-608. CrossRef Medline

Bading H (2017) Therapeutic targeting of the pathological triad of extrasynaptic NMDA receptor signaling in neurodegenerations. J Exp Med 214: 569-578. CrossRef Medline

Bading H, Greenberg ME (1991) Stimulation of protein tyrosine phosphorylation by NMDA receptor activation. Science 253:912-914. CrossRef Medline

Bahari-Javan S, Maddalena A, Kerimoglu C, Wittnam J, Held T, Bähr M, Burkhardt S, Delalle I, Kügler S, Fischer A, Sananbenesi F (2012) HDAC1 regulates fear extinction in mice. J Neurosci 32:5062-5073. CrossRef Medline

Barondes SH, Cohen HD (1968) Memory impairment after subcutaneous injection of acetoxycycloheximide. Science 160:556-557. CrossRef Medline

Bengtson CP, Freitag HE, Weislogel JM, Bading H (2010) Nuclear calcium sensors reveal that repetition of trains of synaptic stimuli boosts nuclear calcium signaling in CA1 pyramidal neurons. Biophys J 99:4066-4077. CrossRef Medline

Bouton ME (2004) Context and behavioral processes in extinction. Learn Mem 11:485-494. CrossRef Medline

Christy B, Nathans D (1989) Functional serum response elements upstream of the growth factor-inducible gene zif268. Mol Cell Biol 9:4889-4895. CrossRef Medline

Cohen H, Kozlovsky N, Matar MA, Zohar J, Kaplan Z (2014) Distinctive hippocampal and amygdalar cytoarchitectural changes underlie specific patterns of behavioral disruption following stress exposure in an animal model of PTSD. Eur Neuropsychopharmacol 24:1925-1944. CrossRef Medline

Fernández E, Jelinek HF (2001) Use of fractal theory in neuroscience: methods, advantages, and potential problems. Methods 24:309-321. CrossRef Medline

Hardingham GE, Chawla S, Johnson CM, Bading H (1997) Distinct functions of nuclear and cytoplasmic calcium in the control of gene expression. Nature 385:260-265. CrossRef Medline

Hawk JD, Bookout AL, Poplawski SG, Bridi M, Rao AJ, Sulewski ME, Kroener BT, Manglesdorf DJ, Abel T (2012) NR4A nuclear receptors support memory enhancement by histone deacetylase inhibitors. J Clin Invest 122:3593-3602. CrossRef Medline

Heldt SA, Stanek L, Chhatwal JP, Ressler KJ (2007) Hippocampus-specific deletion of BDNF in adult mice impairs spatial memory and extinction of aversive memories. Mol Psychiatry 12:656-670. CrossRef Medline

Henze DA, Buzsáki G (2001) Action potential threshold of hippocampal pyramidal cells in vivo is increased by recent spiking activity. Neuroscience 105:121-130. CrossRef Medline

Kassem MS, Lagopoulos J, Stait-Gardner T, Price WS, Chohan TW, Arnold JC, Hatton SN, Bennett MR (2013) Stress-induced grey matter loss determined by MRI is primarily due to loss of dendrites and their synapses. Mol Neurobiol 47:645-661. CrossRef Medline

Katche C, Cammarota M, Medina JH (2013) Molecular signatures and mechanisms of long-lasting memory consolidation and storage. Neurobiol Learn Mem 106:40-47. CrossRef Medline

Longair MH, Baker DA, Armstrong JD (2011) Simple neurite tracer: Open source software for reconstruction, visualization and analysis of neuronal processes. Bioinformatics 27:2453-2454. CrossRef Medline

Mauceri D, Freitag HE, Oliveira AM, Bengtson CP, Bading H (2011) Nuclear calcium-VEGFD signaling controls maintenance of dendrite arborization necessary for memory formation. Neuron 71:117-130. CrossRef Medline

Mauceri D, Hagenston AM, Schramm K, Weiss U, Bading H (2015) Nuclear 
calcium buffering capacity shapes neuronal architecture. J Biol Chem 290:23039-23049. CrossRef Medline

Oliveira AM, Hemstedt TJ, Bading H (2012) Rescue of aging-associated decline in Dnmt3a2 expression restores cognitive abilities. Nat Neurosci 15:1111-1113. CrossRef Medline

Oliveira AM, Hemstedt TJ, Freitag HE, Bading H (2016) Dnmt3a2: a hub for enhancing cognitive functions. Mol Psychiatry 21:1130-1136. CrossRef Medline

Puškaš N, Zaletel I, Stefanović BD, Ristanović D (2015) Fractal dimension of apical dendritic arborization differs in the superficial and the deep pyramidal neurons of the rat cerebral neocortex. Neurosci Lett 589:8891. CrossRef Medline

Ramamoorthi K, Fropf R, Belfort GM, Fitzmaurice HL, McKinney RM, Neve RL, Otto T, Lin Y (2011) Npas4 regulates a transcriptional program in CA3 required for contextual memory formation. Science 334:1669-1675. CrossRef Medline

Sananbenesi F, Fischer A, Wang X, Schrick C, Neve R, Radulovic J, Tsai LH (2007) A hippocampal Cdk5 pathway regulates extinction of contextual fear. Nat Neurosci 10:1012-1019. CrossRef Medline

Schindelin J, Arganda-Carreras I, Frise E, Kaynig V, Longair M, Pietzsch T, Preibisch S, Rueden C, Saalfeld S, Schmid B, Tinevez JY, White DJ, Hartenstein V, Eliceiri K, Tomancak P, Cardona A (2012) Fiji: an open source platform for biological image analysis. Nat Methods 9:676-682. CrossRef Medline

Sholl DA (1953) Dendritic organization in the neurons of the visual and motor cortices of the cat. J Anat 87:387-406.1. Medline

Smith TG Jr, Lange GD, Marks WB (1996) Fractal methods and results in cellular morphology: dimensions, lacunarity and multifractals. J Neurosci Methods 69:123-136. CrossRef Medline

Vianna MR, Szapiro G, McGaugh JL, Medina JH, Izquierdo I (2001) Retrieval of memory for fear-motivated training initiates extinction requiring protein synthesis in the rat hippocampus. Proc Natl Acad Sci U S A 98:12251-12254. CrossRef Medline

von Hertzen LS, Giese KP (2005) Memory reconsolidation engages only a subset of immediate-early genes induced during consolidation. J Neurosci 25:1935-1942. CrossRef Medline

Weislogel JM, Bengtson CP, Müller MK, Hörtzsch JN, Bujard M, Schuster CM, Bading H (2013) Requirement for nuclear calcium signaling in Drosophila long-term memory. Sci Signal 6:ra33. CrossRef Medline

West AE, Greenberg ME (2011) Neuronal activity-regulated gene transcription in synapse development and cognitive function. Cold Spring Harb Perspect Biol 3: pii: a005744. CrossRef Medline

Yehuda R, LeDoux J (2007) Response variation following trauma: a translational neuroscience approach to understanding PTSD. Neuron 56:1932. CrossRef Medline

Zhang SJ, Steijaert MN, Lau D, Schütz G, Delucinge-Vivier C, Descombes P, Bading H (2007) Decoding NMDA receptor signaling: identification of genomic programs specifying neuronal survival and death. Neuron 53: 549-562. CrossRef Medline

Zhang SJ, Zou M, Lu L, Lau D, Ditzel DAW, Delucinge-Vivier C, Aso Y, Descombes P, Bading H (2009) Nuclear calcium signaling controls expression of a large gene pool: Identification of a gene program for acquired neuroprotection induced by synaptic activity. PLoS Genet 5 . e1000604. 